\title{
Measuring cluster gas mass fractions with X-ray, Sunyaev-Zel'dovich effect, and weak lensing data
}

\author{
S. LaRoque ${ }^{1}$, M. Bonamente ${ }^{2}$, J. E. Carlstrom ${ }^{1,3}$, \\ M. Joy ${ }^{4}$ and E. D. Reese ${ }^{5}$ \\ ${ }^{1}$ Department of Astronomy and Astrophysics, University of Chicago, Chicago, IL 60637 \\ ${ }^{2}$ Department of Physics, University of Alabama, Huntsville, AL 35899 \\ ${ }^{3}$ Kavli Institute for Cosmological Physics, Department of Physics, Enrico Fermi Institute, \\ University of Chicago, Chicago, IL 60637 \\ ${ }^{4}$ Department of Space Science, NASA Marshall Space Flight Center, Huntsville, AL 35812 \\ ${ }^{5}$ Department of Physics, University of California, Berkeley, CA 94720
}

\begin{abstract}
We estimate the gas mass fractions for a sample of 39 high- $z$ galaxy clusters using a combination of three different data sets: Chandra X-ray data, OVRO/BIMA centimeter-wave Sunyaev-Zel'dovich Effect (SZE) data, and published weak gravitational lensing data. The gas mass fraction $f_{g}$ is calculated in three different ways. The X-ray surface brightness and SZE decrement are used independently to determine the number of baryons in the intracluster gas and subsequently the gas mass; the total mass follows assuming spherical symmetry and hydrostatic equilibrium (HSE). The third, less model-dependent approach is to calculate the projected SZE gas mass along a cylinder and divide by the total mass derived from the lensing data. The goal of this study is to compare the $f_{g}$ results from the three different methods to investigate systematic uncertainties associated with clumping of the intracluster medium, cluster elongation, and the HSE assumption.
\end{abstract}

\section{Introduction}

Clusters of galaxies are the largest known virialized objects and can be used as cosmological probes. The mass composition on cluster scales is expected to reflect the universal mass composition; clusters are therefore a useful probe of $\Omega_{B} / \Omega_{M}$. In this preliminary analysis, we have calculated the gas mass fraction $f_{g}$ for a sample of 39 clusters $(0.14 \leqslant z \leqslant 0.89)$ observed by both the Chandra X-ray observatory and the BIMA/OVRO interferometric Sunyaev-Zel'dovich Effect (SZE) imaging experiment. The $f_{g}$ calculation is performed in two parallel ways, by studying the cluster's X-ray emission and its SZE. We have also combined SZE data with weak gravitational lensing data to calculate $f_{g}$ a third way for fifteen of the clusters in our sample. Due to the differing dependencies of these methods on the cluster properties, the results can be compared to better understand systematics associated with assumptions about clumping of the intracluster medium, temperature structure, cluster morphology, and hydrostatic equilibrium (HSE). A $\Lambda$ CDM cosmology with $\Omega_{M}=0.3$ and $\Omega_{\Lambda}=0.7$ is assumed throughout and $H_{0}=70 \mathrm{~km} \mathrm{~s}^{-1} \mathrm{Mpc}^{-1}$. All uncertainties are at the $90 \%$ level. 


\section{Data analysis}

\subsection{X-ray data}

Clusters were observed with the Chandra ACIS detector. Seven clusters in the sample are seen to have "cooling flow" cores; their bright central regions were excised prior to spatial and spectral fitting. Electron temperatures are calculated by fitting the $0.7-7.0 \mathrm{keV}$ spectral data to a Raymond-Smith thermal plasma model. The X-ray surface brightness $S_{x}$ is fit to an isothermal spherical $\beta$-model, and the resulting density distribution is used to get the gas mass as a function of radius. Care is taken not to extrapolate the model beyond the region where X-ray emission is detected. The total gravitational mass is determined assuming HSE, and the cluster gas mass fraction is then simply $f_{g}=$ $M_{\text {gas }} / M_{\text {tot }}$. A Markov chain Monte-Carlo (MCMC) method is used to determine the probability distributions and best-fit values of the model parameters and masses. Gas mass fractions are reported within $r_{2500}$, the radius at which the mean mass density of the cluster is equal to 2500 times the critical density, $\rho_{c}(z)$, at that epoch.

\subsection{SZE data}

SZE observations were conducted at the Berkeley Illinois Maryland Association (BIMA) and Owens Valley Radio Observatory (OVRO) interferometric arrays at $28.5 \mathrm{GHz}$ (BIMA and OVRO) and at $30 \mathrm{GHz}(\mathrm{OVRO})$. The X-ray derived $T_{e}$ is assumed, and the SZE brightness profile is fit to an isothermal spherical $\beta$-model. The density distribution is then used to get the gas mass. As before, the total masses are calculated under the assumption of HSE. A MCMC is used to determine probability distributions for the model parameters and masses. The masses are best constrained at a radius of $65 "$ (Grego et al. 2001) and results are reported there.

\subsection{Lensing data}

The gas mass fraction is calculated in a third way for fifteen of the clusters by comparing the SZE-derived gas masses with the total masses determined from weak lensing data. Lensing masses come from Clowe et al. (1998) and Dahle et al. (2002). Gravitational lensing and the SZE both probe a cluster's projected mass distribution within a cylinder whose long axis is the line of sight. No deprojection is therefore necessary to calculate $f_{g}$. Comparison of the results from all three methods should illuminate any trends in cluster elongation, and the $T_{e}$ independence of the lensing masses should help constrain temperature structure and non-thermal pressure support. Using the SZE-derived $\beta$-model parameters, we calculate the gas mass in a cylindrical volume of radius 65 " and compare it with the gravitational lensing mass at the same radius to get $f_{g}$. Since the published lensing masses are calculated assuming a flat universe with $\Lambda=0$, the cylindrical SZE gas masses are calculated assuming the same cosmology. A rough scaling is then applied to make the SZE/lensing values consistent with the $\Lambda \mathrm{CDM}$ cosmology assumed for the SZE and X-ray gas mass fractions.

\section{Results}

The X-ray, SZE, and SZE/lensing gas mass fractions are shown in Figure 1. Uncertainties are statistical only. There is no evidence of evolution with redshift for this cosmology, and the gas mass fractions for individual clusters agree reasonably well within the uncertainties. There are possible trends apparent in the ensemble averages, however. We have calculated mean gas mass fractions for the three datasets in log space, where the uncertainties are nearly Gaussian (cf. Figure 1). We find $\bar{f}_{g}=0.094 \pm 0.002$ for the Xray data, $\bar{f}_{g}=0.079_{-0.007}^{+0.010}$ for the SZE data, and $\bar{f}_{g}=0.075_{-0.011}^{+0.017}$ for the SZE/lensing 


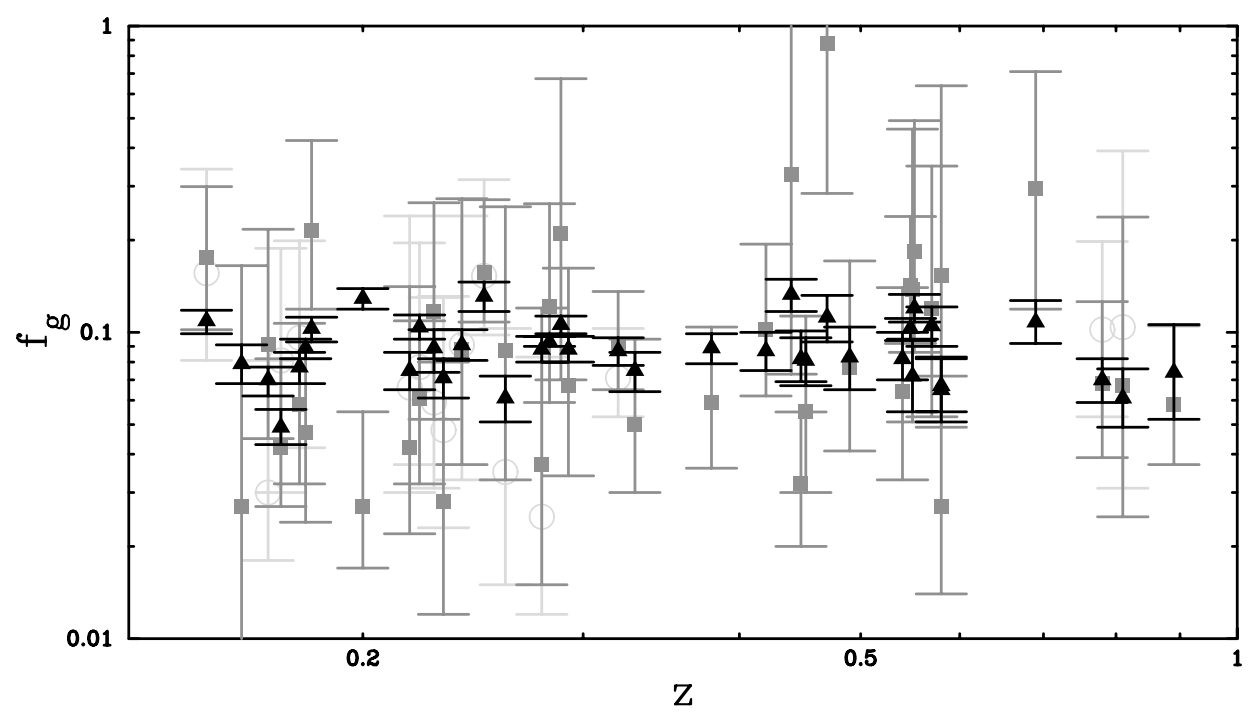

Figure 1. X-ray (black triangles), SZE (dark grey squares), and SZE/lensing (light grey circles) gas mass fractions versus redshift.

analysis. The good agreement between the SZE and SZE/lensing results is encouraging and suggests that the HSE assumption is appropriate, but the offset relative to the X-ray value needs to be explored. Clumping of the intracluster medium may bias the X-ray gas fractions high, while the isothermal assumption may bias the SZE results low, since $T_{e}$ as measured from the X-ray data is an $n_{e}^{2}$-weighted value. Furthermore, the assumption of a flat gas mass fraction profile may cause the SZE gas mass fractions to be lower than the X-ray values, since $65^{\prime \prime}$ is generally comparable to or less than $r_{2500}$. These are preliminary results from a work in progress, and a forthcoming study will incorporate more realistic temperature profiles, SZE gas fractions scaled with radius, and insights from a concurrent cluster distance project.

\section{Acknowledgements}

This material is based upon work supported by NSF grant No. 0096913 and by NASA LTSA grant NAG5-7986. S. L. gratefully acknowledges support from NASA GSRP Fellowship NGT5-50173.

\section{References}

Clowe, D., Luppino, G., Kaiser, N., Henry, I. \& Gioia, I. 1998 ApJ 497, L61-L64.

Dahle, H., Kaiser, N., Irgens, R., Lilje, P., \& Maddox, S. 2002 ApJS 139, 313-368.

Grego, L., Carlstrom, J., Reese, E., Holder, G., Holzapfel, W., Joy, M., Mohr, J., \& Patel, S. 2001 ApJ 552, 2-14 


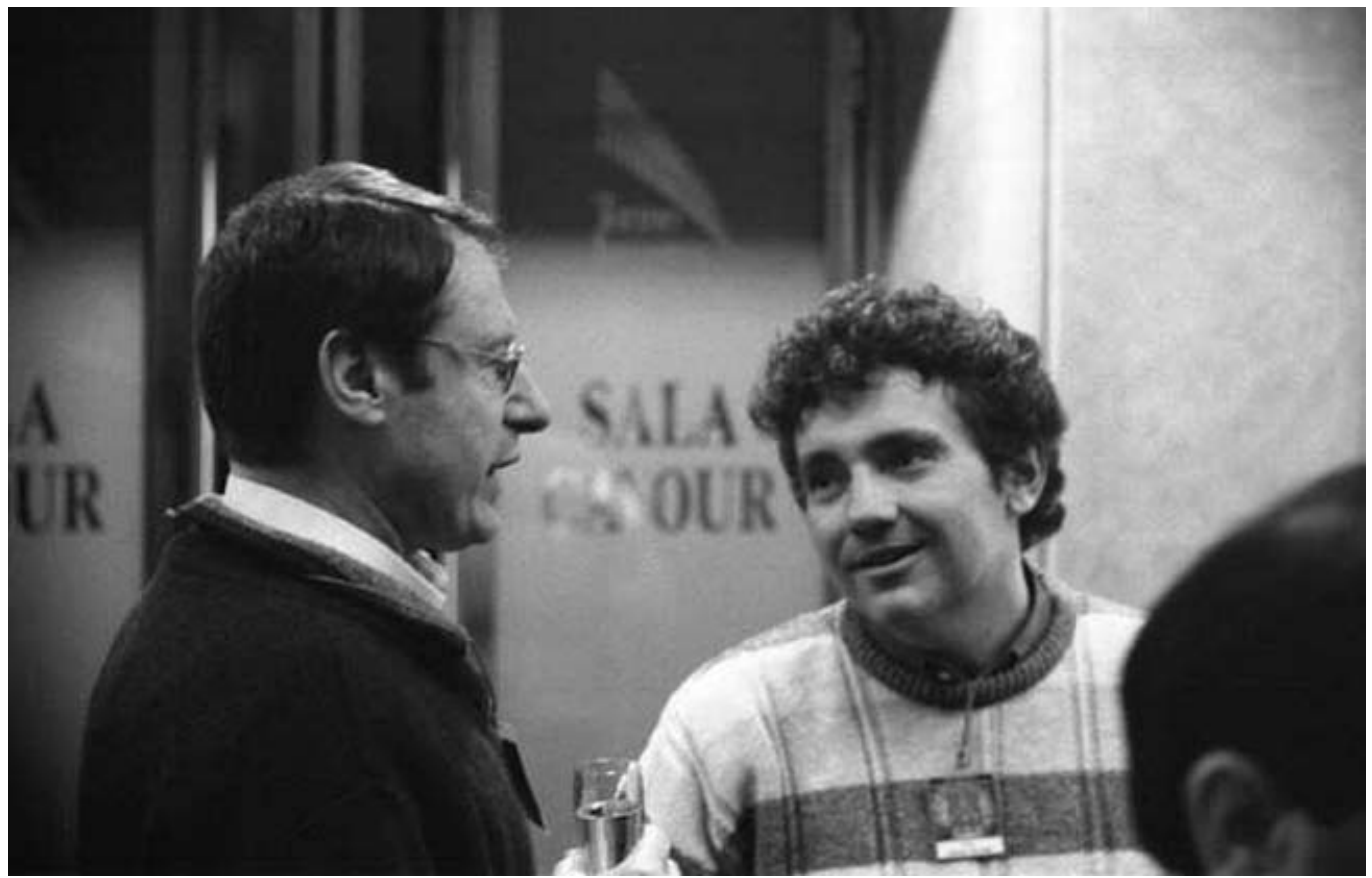

Hans Böhringer explains to Sandro Bardelli how relevant white wine is to understand cosmology (by J.M. Colberg).

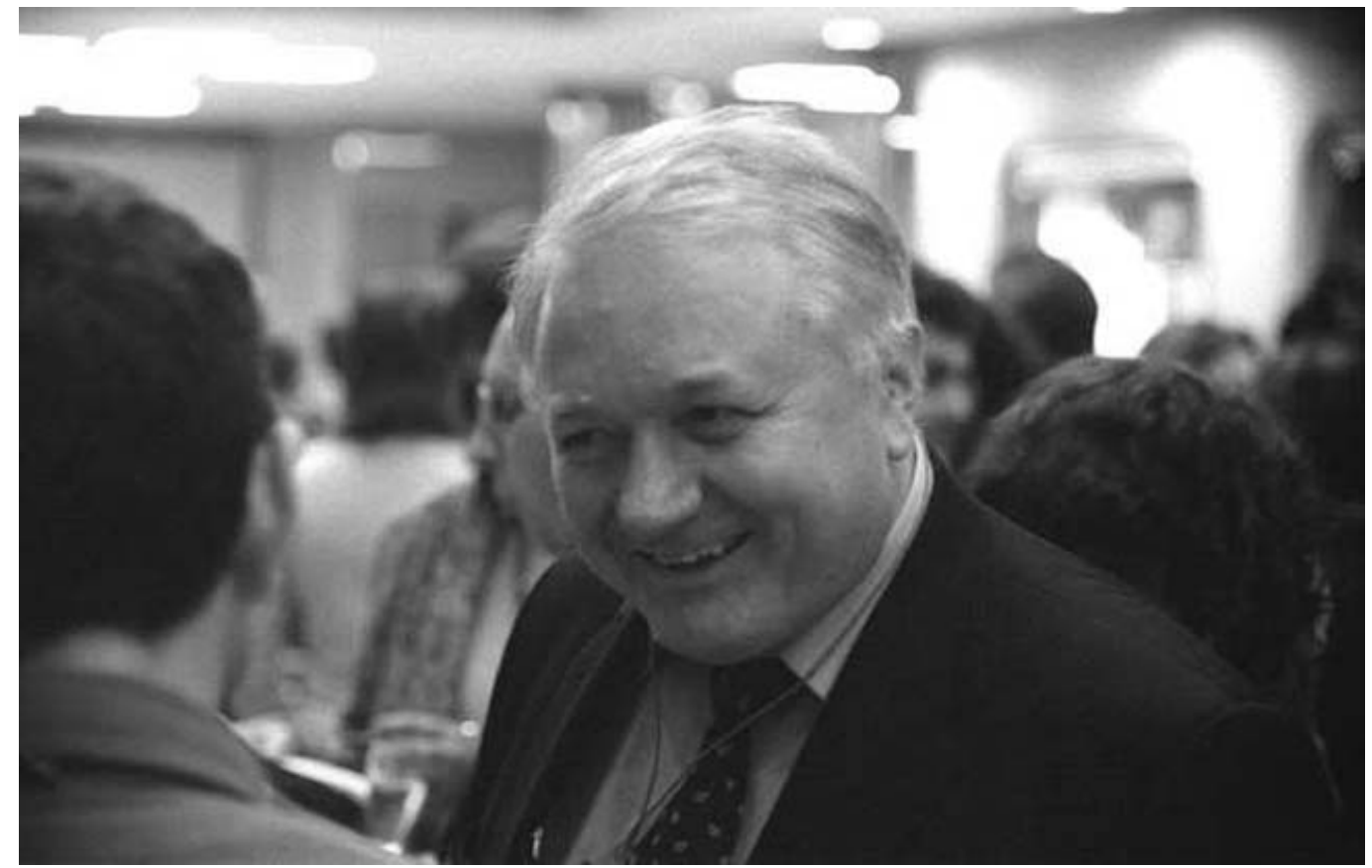

Rashid Sunyaev enjoys the discussion at the welcome party (by J.M. Colberg). 